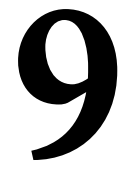

\title{
LECTURA, OCIO Y RENDIMIENTO ACADÉMICO EN ESTUDIANTES UNIVERSITARIOS DEL ÁREA SOCIOEDUCATIVA
}

\section{(READING, LEISURE AND ACADEMIC PERFORMANCE IN UNIVERSITY STUDENTS OF THE SOCIOEDUCATIONAL AREA)}

María Elche

Sandra Sánchez-García

Santiago Yubero

Universidad de Castilla-La Mancha

DOI: $10.5944 / e d u c X X 1.21548$

Cómo referenciar este artículo/How to reference this article:

Elche, M.; Sánchez-García, S. y Yubero, S. (2019). Lectura, ocio y rendimiento académico en estudiantes universitarios del área socioeducativa. Educación XX1, 22(1), 215-237, doi: 10.5944/ educXX1.21548

Elche, M.; Sánchez-García, S. \& Yubero, S. (2019). Lectura, ocio y rendimiento académico en estudiantes universitarios del área socioeducativa. [Reading, leisure and academic performance in university students of the socioeducational area]. Educación XX1, 22(1), 215-237, doi: 10.5944/ educXX1.21548

\section{RESUMEN}

Introducción: En los últimos años se ha potenciado el interés de la investigación en torno a las variables que determinan el éxito académico dentro del ámbito universitario. Diferentes estudios han analizado el papel de la lectura en el rendimiento, pero, sin embargo, no se ha considerado la posible contribución conjunta respecto al ocio en el éxito académico de los universitarios. Método: El objetivo de esta investigación es analizar cómo se inserta la lectura voluntaria en el tiempo de ocio de los estudiantes universitarios y estudiar la influencia de la relación del ocio y de la lectura con el éxito académico en la universidad. Se ha empleado el Cuestionario de Hábitos de Lectura, la Escala de Actividades de Tiempo Libre y una medida del rendimiento. El cuestionario fue completado por 1.614 estudiantes universitarios. Resultados: Los resultados confirmaron la relación del 
rendimiento académico con el ocio cultural junto al tecnológico y con las prácticas de lectura, especialmente de tipo profesional. Discusión: Sería necesario seguir profundizando en esta línea de investigación con el fin de diseñar propuestas de intervención en la universidad centradas en la mejora de habilidades de lectura y en la gestión del tiempo de ocio.

\section{PALABRAS CLAVE}

Educación superior; estudiantes universitarios; hábitos de lectura; tiempo de ocio; rendimiento académico.

\section{ABSTRACT}

Introduction: In recent years, the interest of research has been strengthened around the variables that determine academic success within the university context. Different studies have analyzed the role of reading in achievement, but, nevertheless, the possible joint contribution with respect to leisure in the academic success of university students has not been considered. Methodology: The aim of this research is to analyze how voluntary reading is inserted in the leisure time of university students and to study the influence of the relationship of leisure and reading with academic success in university. The Reading Habits Questionnaire, the Leisure Activity Scale and one academic achievement measure have been used. The questionnaire was completed by 1,614 university students. Results: The results confirmed the relationship between academic achievement and cultural and technological leisure, and with reading practices, particularly of a professional nature. Discussion: It would be necessary to continue to examine this line of research in order to design proposals for intervention at the university focused on improving reading skills and leisure time management.

\section{KEYWORDS}

University studies; reading; leisure; achievement. 


\section{INTRODUCCIÓN}

La calidad formativa de los estudiantes universitarios debe asegurar su inclusión en un mundo constantemente en evolución. En este sentido, la universidad tiene que facilitar las herramientas para gestionar los conocimientos que garanticen la actualización continua de los mismos. La lectura es la base por la que se adquieren los aprendizajes. Además, la lectura voluntaria, la que se realiza libremente durante el tiempo de ocio, potencia las capacidades cognitivas precisas para cualquier estudiante universitario, tanto durante la carrera como en su desarrollo profesional: percibir, asimilar, interpretar, resumir, contrastar, asentir, disentir y criticar (Sánchez-Chévez, 2012). Por otra parte, la identificación de las buenas prácticas de ocio se hace cada vez más relevante para la intervención en la mejora de la calidad de vida y el desarrollo integral de los jóvenes (PozaVilches, Fernández-García, y Ferreira-Delgado, 2018). Como han indicado otros autores, es preciso conocer qué implicación tiene el empleo del tiempo libre sobre la vida académica (Bermúdez, Ramírez, y Jaramillo, 2016) y, específicamente, analizar el papel que ocupa la lectura voluntaria en el éxito académico universitario.

La investigación en torno a las variables que determinan el éxito académico en la escuela es un clásico entre los estudios de la educación. Su interés dentro del ámbito universitario se ha potenciado en los últimos años (Belvis, Moreno, y Ferrer, 2009; García-Ros y Pérez-González, 2011; Tejedor y García-Valcárcel, 2007). El análisis de las potencialidades del alumnado, frente al enfoque del déficit, ha experimentado un elevado auge dentro de los aspectos educativos (Froh, Huebner, Youssef, \& Conte, 2011). Una línea de trabajo sobre el éxito académico se ha centrado en el análisis de las variables personales: sexo, tiempo de dedicación, estatus económico y sociofamiliar (Kitsantas, Winsler \& Huie, 2008; Lassibille y Navarro, 2007). Una segunda línea se centra en las características psicosociales de los alumnos: compromiso con los estudios, autoeficacia, apoyo social, gestión del tiempo y hábitos de estudio (Bethencourt, Cabrera, Hernández, Álvarez, y González, 2008; García-Ros y Pérez-González, 2011). En esta línea psicosocial es donde se ubica este trabajo, cuyo objetivo fundamental es determinar la influencia del ocio y de la lectura voluntaria sobre el rendimiento académico universitario.

\section{Ocio y tiempo libre}

Son numerosos los trabajos que tratan el tiempo libre y su vivencia como ocio. Según Rodríguez-Suárez y Agulló (1999), «el ocio será, dentro del tiempo libre, aquel conformado por las actividades y prácticas 
libremente elegidas según las preferencias de cada individuo»(p. 249). Dumazedier (1971) ya indicó que «el ocio es un conjunto de ocupaciones a las que el individuo se entrega de manera completamente voluntaria... para desarrollar su información o formación desinteresada, su participación social...» (p. 20). En la misma línea, Caride (2014) define el ocio como un ámbito de desarrollo humano en el que resulta determinante la vivencia de libertad y sensación placentera, de manera que «lo importante en el tiempo de ocio no son tanto las actividades que realizar como la significación que estas tienen para las personas, su posible provecho para la formación personal y el desarrollo social» (p. 41).

La investigación sobre el ocio se intensificó en la segunda mitad del siglo Xx, entendiéndolo como un bien logrado por nuestra sociedad y necesario para el buen equilibrio personal. No solo por su función evasiva, sino por su importancia en la producción de relaciones, intercambio de normas, conductas y valores (Centro Nacional de Información y Comunicación Educativa [CNICE], 2007) y por su papel en el crecimiento y desarrollo personal (García-Cué y Santizo, 2010). Se destaca su trascendencia para cualquier desarrollo humano que se pretenda, integral e integrador, de todas las personas y del todo en cada persona: individual y colectivo, cognitivo y emocional (Caride, 1998; Cuenca, 2009). Así, en el conjunto de los denominados tiempos sociales, el ocio emerge como una necesidad y un derecho cívico, del que cabe esperar una contribución a la mejora del bienestar de las personas y de su calidad de vida (Caride, 2012).

En la sociedad que inicia el siglo XXI, el tiempo de ocio ha pasado de ser una atractiva posibilidad a constituirse como un derecho en sí mismo (Caride, 2009), siendo estimado en mayor medida por el colectivo juvenil (Aristegui y Silvestre, 2012). Se ha transformado el papel y las vivencias de ocio, se modifican determinadas tendencias y se abren espacios para reivindicar un ocio menos consumista y más facilitador de experiencias que puedan derivar de un ocio valioso (Cuenca, 2013).

Cuenca y Goytia (2012) revelan que la sociedad actual concede más oportunidades para la realización de experiencias de ocio, si bien cada individuo las usa de forma diferente dependiendo de sus actitudes, intereses, competencias y capacidad de disfrute; cuestiones, según los autores, más relacionadas con su formación que con la oferta. En relación con esto, un estudio de Rodríguez-Suárez y Agulló (1999) revela que existe una relación en los jóvenes que cursan estudios superiores con el tiempo de prácticas culturales, de ocio y tiempo libre. Según indican, es más significativo el componente cultural en estas actividades en los universitarios que en las realizadas por otros colectivos juveniles, por lo que entienden que 
... los jóvenes universitarios forman un grupo diferenciado del resto de la juventud, no solo por la franja de edad en que están comprendidos, sino también... por su estatus económico y social, así como por el itinerario formativo que están recorriendo (y el capital formativo que supone esta trayectoria) y porque supuestamente integran un grupo con estilo de vida propio frente al resto de jóvenes ... (p. 248)

Existen investigaciones que muestran evidencias sobre la relación existente entre determinadas conductas de ocio en relación con la habilidad de estudio y, en consecuencia, con el rendimiento académico (Fabiano, Mckinney, Rhoads, y Starks, 2000). Tirado, Aguaded y Marín (2010) realizaron un estudio para identificar diferentes perfiles de estudiantes universitarios sobre la base de sus hábitos de ocupación del tiempo de ocio y comprobar su efecto sobre el hábito de estudio. Los resultados mostraron indicios para pensar en la influencia positiva de la afición a la lectura y al deporte sobre la frecuencia de estudio, así como la influencia negativa del consumo de drogas.

\section{Lectura y rendimiento}

Conseguir una buena comprensión y competencia lectora es uno de los principales objetivos educativos y un prerrequisito cada vez más esencial para alcanzar el éxito en las sociedades actuales (Clark \& Rumbold, 2006). En el informe de la Organización de las Naciones Unidas para la Educación, la Ciencia y la Cultura (UNESCO), coordinado por Delors (1997), se afirmaba que los libros y la lectura constituyen los pilares básicos de la educación y de la difusión del conocimiento. La misma idea fue recogida en el informe del Programme for International Student Assessment (PISA) (Organización para la Cooperación y el Desarrollo Económicos [OCDE], 2012), que definió la lectura como una competencia fundamental dentro y fuera del ámbito escolar, que ayuda a asumir el pensamiento y participar en la sociedad. La lectura ha adquirido gran importancia como herramienta de educación y acceso a la información (Dezcallar, Clariana, Cladellas, Badia, y Gotzen, 2014) y se ha constituido como la práctica cultural con mayor reconocimiento, debido a su papel como instrumento para el desarrollo de la mentalidad crítica (Fernández, 2005).

Diversos estudios han mostrado que la capacidad lectora está vinculada con el éxito en los aprendizajes desde la escuela a la universidad (Clerici, Monteverde, y Fernández, 2015; Lotti-de-Santos, Salim, Raya, y Dori 2008; Noursi, 2014; Whitten, Labby, \& Sullivan, 2016; Yubero, Larrañaga, y Sánchez-García, 2011). Se han encontrado relaciones significativas entre las calificaciones escolares y variables tales como el gusto por la lectura 
(Molina, 2006) o la comprensión lectora (Fiz, Goicoechea, Ibiricu, y Olea, 2000). En estudiantes adolescentes, Mol y Jolles (2014) sugieren que se produce una espiral entre el placer por la lectura, la lectura voluntaria y el rendimiento. Los no lectores presentan riesgo de peor rendimiento, mientras que los estudiantes que leen de forma voluntaria tienen mayor desarrollo de mapas mentales, lo que facilita su continuidad lectora y el éxito académico. Un estudio de Gil-Flores (2011) mostró que los mayores niveles de desarrollo en las competencias básicas del alumnado se registran cuando los sujetos poseen el hábito de leer como forma de experiencia literaria, dirigida fundamentalmente al disfrute personal. Según este autor, el hábito lector propicia una mejora de la competencia lectora y otras habilidades cognitivas que están en la base y son el fundamento para un aprendizaje continuo a lo largo de toda la vida. El informe PISA 2011 (OCDE, 2012) lo confirmó, concluyendo que aquellos jóvenes que diariamente leen por placer obtienen mejores rendimientos en la prueba.

La lectura pasa a formar parte esencial del contexto universitario, siendo una parte importante del núcleo de la vida académica, como vía de acceso a la información y como elemento imprescindible para la adquisición de competencias (Carlino, 2013). La importancia radica en que, como afirman Vidal y Manríquez (2016), la lectura, en cuanto a comprensión y producción de información, también es un eje fundamental en la cognición de los universitarios. En la investigación de Moyano (2004) los estudiantes universitarios lectores tienen mejor desarrollo en su disciplina y una mejor inserción en la actividad académica. Otros estudios centran la participación académica en la mayor intervención en las sesiones de discusión desarrolladas en las clases (Lei, Bartlett, Gorney \& Herschbach, 2010) y con intervenciones más estimulantes (Ruscio, 2001). También están más motivados en las clases y planifican mejor las actividades de aprendizaje (Hoeft, 2012), lo que parece facilitar un rendimiento superior en la universidad (Galicia y Villuendas, 2011). Por el contrario, los estudiantes universitarios no lectores son solamente receptores, presentan menor rendimiento, poca participación en las discusiones de clase, actitud pasiva en el aula y poca disposición hacia la investigación (Torres, 2003).

Como vemos, diferentes estudios han analizado el papel de la lectura en el rendimiento pero, sin embargo, no se ha considerado la posible contribución conjunta respecto al ocio en el éxito académico de los universitarios. Esta investigación analiza, desde la perspectiva psicosocial, cómo influyen las actividades de ocio, entre ellas especialmente la lectura voluntaria, sobre el rendimiento de los estudiantes universitarios. El objetivo de este trabajo ha sido estudiar la vinculación conjunta del ocio y de la lectura sobre el éxito académico universitario de los estudiantes del ámbito socioeducativo; concretamente, cómo se inserta la lectura voluntaria en el tiempo de ocio de 
estos y estudiar su influencia conjunta en el ámbito de la universidad. Este objetivo general se desglosa en los siguientes objetivos específicos:

- Analizar la influencia del ocio sobre el rendimiento.

- Analizar la influencia de los hábitos lectores sobre el rendimiento.

- Conocer la influencia conjunta de ambas variables a partir del estudio del perfil de ocio y lectura que caracteriza a los estudiantes del área socioeducativa con alto rendimiento universitario.

\section{MÉTODO}

\section{Participantes}

La muestra quedó constituida por 1614 estudiantes universitarios de las universidades de Castilla-La Mancha y Santiago de Compostela, con una edad comprendida entre 18 y 52 años, siendo un 90\% menor de 25 años $(M=21.28, D T=3.36)$. Según el grado del que proceden, se distribuyen de la siguiente manera: $25.8 \%$ de Educación Social, 9.5\% de Trabajo Social, $21.7 \%$ de Educación Infantil, 31.4\% de Educación Primaria y 11.6\% de Pedagogía. Respecto al sexo, la muestra está sesgada por la procedencia de los grados; un $16.5 \%$ son hombres y un $83.3 \%$ mujeres. En la tabla 1 se muestran las características de los participantes según la universidad de procedencia.

Tabla 1

Descripción de la muestra

\begin{tabular}{l|cc}
\hline \multirow{2}{*}{ Variable } & \multicolumn{2}{|c}{ Universidad n (\%) } \\
\cline { 2 - 3 } Sexo & Castilla-La Mancha & Santiago de Compostela \\
Hombre & $171(19.3)$ & $95(13.0)$ \\
Mujer & $710(80.7)$ & $638(87.0)$ \\
\hline Grado & & $179(24.4)$ \\
Educación Social & $237(26.9)$ & - \\
Trabajo Social & $153(17.4)$ & $158(21.6)$ \\
Educación Infantil & $193(21.9)$ & $208(28.4)$ \\
Educación Primaria & $298(33.8)$ & $188(25.6)$ \\
Pedagogía & 0 & $187(24.4)$ \\
\hline Curso & $345(39.2)$ & $182(24.8)$ \\
1. & $345(39.2)$ & $220(30.0)$ \\
. $^{\circ}$ & $146(16.6)$ & $144(19.6)$ \\
3. $^{\circ}$ & $45(5.1)$ & $733(45.4)$ \\
4. & $881(54.6)$ & \\
\hline
\end{tabular}




\section{Instrumentos}

Para evaluar el comportamiento lector se seleccionaron las dos preguntas para la construcción del hábito lector del Cuestionario de Hábitos de Lectura (Larrañaga, 2004; Larrañaga y Yubero, 2005), que ya había sido utilizado en estudiantes universitarios (Yubero y Larrañaga, 2015; Yubero, Larrañaga, y Pires, 2014). Una pregunta está referida a la frecuencia de lectura voluntaria (nunca, casi nunca, al menos una vez al trimestre, al menos una vez al mes, al menos una vez a la semana y todos o casi todos los días) y la otra al número de libros leídos por placer durante el último año (ninguno, 1 o 2 , de 3 a 5 , de 6 a 10 , de 11 a 15 , de 15 a 20 , de 21 a 50 o más de 50). El cuestionario alcanzó una fiabilidad de $\alpha=.77$.

En investigaciones previas (Larrañaga y Yubero, 2005), se ha informado que en los resultados del cruce de respuestas entre ambas variables aparecían resultados inconsistentes. Se encontraban sujetos que informaban de comportamiento lector en una variable y de comportamiento no lector en la otra; parecía que algunos estudiantes querían ofrecer una imagen diferente a la real. Cruzamos ambas variables para construir el hábito lector. Solo hemos considerado 'lector habitual' a los sujetos que responden en esta categoría en las dos variables. Consideramos como 'lector ocasional' a los sujetos en los que se produce el cruce de dos valoraciones de lector ocasional y el cruce de lector habitual con lector ocasional. Entra en la categoría 'no lector' el cruce de no lector en ambas categorías. La combinación de 'no lector' en cualquiera de las dos variables con una valoración de lector (ocasional o habitual) en la otra lo hemos designado como sujeto 'falso lector', por entender que realmente es no lector, pero está queriendo dar una imagen lectora. De esta manera, se obtienen cuatro grupos de lectores:

- No lectores: no leen nunca o casi nunca. Entre 0 y 2 libros al año.

- Lectores ocasionales: leen alguna vez al trimestre o al mes. Entre 3 y 10 libros al año.

- Lectores frecuentes: leen casi todos los días o más de una vez a la semana. Más de 10 libros al año.

- Falsos lectores: aquellos que en una variable se muestran como lectores y en la otra como no lectores.

También se solicitó información sobre el interés lector de tres tipologías: literaria, de actualidad y profesional. En los tres casos se medía con una escala de 1 a 10 puntos.

Para obtener la información sobre el ocio se empleó la Escala de Actividades de Tiempo Libre (Yubero et al., 2014), que mide el tiempo 
dedicado a distintas alternativas de ocio: ir al cine, hacer deporte, salir de paseo o a ver tiendas, tomar cañas/copas con los amigos, bailar en discotecas o similares, leer un libro, ver programas de TV, escuchar música o la radio, estar con el ordenador/Internet, no hacer nada, estudiar, leer la prensa o revistas, quedar en casa de los amigos. La información se recoge con una escala de respuesta tipo Likert de cinco anclajes, 1 - Nada, 2- Poco, 3- Algo, 4- Bastante, 5- Mucho. Se incluyó también la medida sobre el tiempo libre disponible durante la semana y el fin de semana (1- Nada, 2- Poco, 3- Algo, 4- Bastante, 5- Mucho) y la satisfacción con su tiempo de ocio, desde 1Totalmente insatisfecho hasta 5- Totalmente satisfecho.

El rendimiento fue medido a través de una pregunta directa sobre su nota media en la universidad, desde 1- suspenso a 4-sobresaliente.

\section{PROCEDIMIENTO}

Los/las participantes fueron seleccionados aleatoriamente, tomando la clase como unidad de muestreo. Los cuestionarios se pasaron en el aula de clase de las distintas universidades, previa autorización de los profesores colaboradores, en un tiempo de entre 10 y 15 minutos. La participación fue voluntaria y anónima, previo consentimiento de los estudiantes, y se aseguró la confidencialidad.

\section{ANÁLISIS DE DATOS}

En primer lugar, se realizó un análisis factorial de las actividades de ocio, usando rotación Varimax, para construir los factores de ocio; posteriormente se realizó un análisis univariante para conocer su influencia sobre el rendimiento académico. También se han analizado las diferencias en rendimiento según el hábito lector a través de un análisis de contingencia y la influencia del interés lector con Anovas. Por último, se han analizado las diferencias en las variables de estudio a través de Anovas, para conocer el perfil de ocio y lectura del estudiante universitario con rendimiento alto.

\section{RESULTADOS}

Para analizar la estructura factorial de las actividades de ocio se realizó un análisis factorial de componentes principales con rotación Varimax. Se ha incluido el ítem en el factor cuando el peso factorial es superior a .300. 
El primer factor estuvo compuesto por cuatro actividades que se corresponden con las salidas con los amigos; es el factor de ocio social. El segundo factor, ocio tecnológico, ver programas de televisión y pasar el tiempo en Internet y/o con el ordenador. El tercer factor quedó constituido con cuatro actividades que se corresponden con el ocio cultural. El peso mayor del ocio cultural recae sobre la lectura en el tiempo libre. En los últimos factores entra solamente una actividad; no hacer nada en el cuarto y deporte en el quinto. La actividad de escuchar música o la radio entra a formar parte de los cuatro últimos factores (todos los que no se relacionan con la interacción entre iguales). La actividad de estudiar no entró en ningún factor (tabla 2). Los resultados descriptivos de los factores mostraron que los estudiantes dedican más tiempo de ocio a estar con los amigos (ocio social, $M=3.14, D T=0.75$ ) y conectados al ordenador o viendo programas de televisión (ocio tecnológico, $M=3.14$, $D T=0.92)$. En medida similar al ocio cultural $(M=2.68, D T=0.65)$ y al deporte $(M=2.69, D T=1.25)$, en menor medida a no hacer nada $(M=2.23, D T=1.13)$.

Tabla 2

Análisis factorial de las actividades de ocio

\begin{tabular}{lcccccc}
\hline & Comunalidad & $\begin{array}{c}\text { Factor } \\
\text { I }\end{array}$ & $\begin{array}{c}\text { Factor } \\
\text { II }\end{array}$ & $\begin{array}{c}\text { Factor } \\
\text { III }\end{array}$ & $\begin{array}{c}\text { Factor } \\
\text { IV }\end{array}$ & $\begin{array}{c}\text { Factor } \\
\text { V }\end{array}$ \\
\hline Discoteca & .715 & .819 & & & & \\
Cañas & .656 & .785 & & & & \\
Casa amigos & .438 & .601 & & & & \\
Paseo & .485 & .525 & & & & \\
TV & .572 & & .770 & & & \\
Ordenador & .578 & & .676 & & & \\
Lectura & .358 & & & .723 & & \\
Prensa & .448 & & & .537 & & \\
Cine & .795 & & .582 & .424 & .310 & .318 \\
Música & .617 & & .382 & & & \\
Estudiar & .709 & & & & .708 & \\
Nada & .668 & & & & & .845 \\
Deporte & .631 & & & & \\
\hline Varianza explicada $(\%)$ & 15.64 & 11.27 & 10.89 & 9.12 & 8.73 \\
\hline
\end{tabular}

Si tenemos en cuenta que el patrón de ocio individual se define por el conjunto de los factores de ocio, por lo que no se puede analizar independientemente cada uno de ellos, se ha llevado a cabo el análisis de la relación del ocio sobre el rendimiento con un análisis univariante incluyendo simultáneamente los cinco factores de ocio y su interacción. El modelo alcanzó significación, $F=7.29, p<.001, \eta^{2}=.19$. Los resultados indican que el ocio cultural solamente incide positivamente sobre el rendimiento 
junto con el ocio tecnológico, pero con un tamaño del efecto muy reducido, $F=2.75, p<.05, \eta^{2}=.012$. En la gráfica 1 puede observarse la interacción.
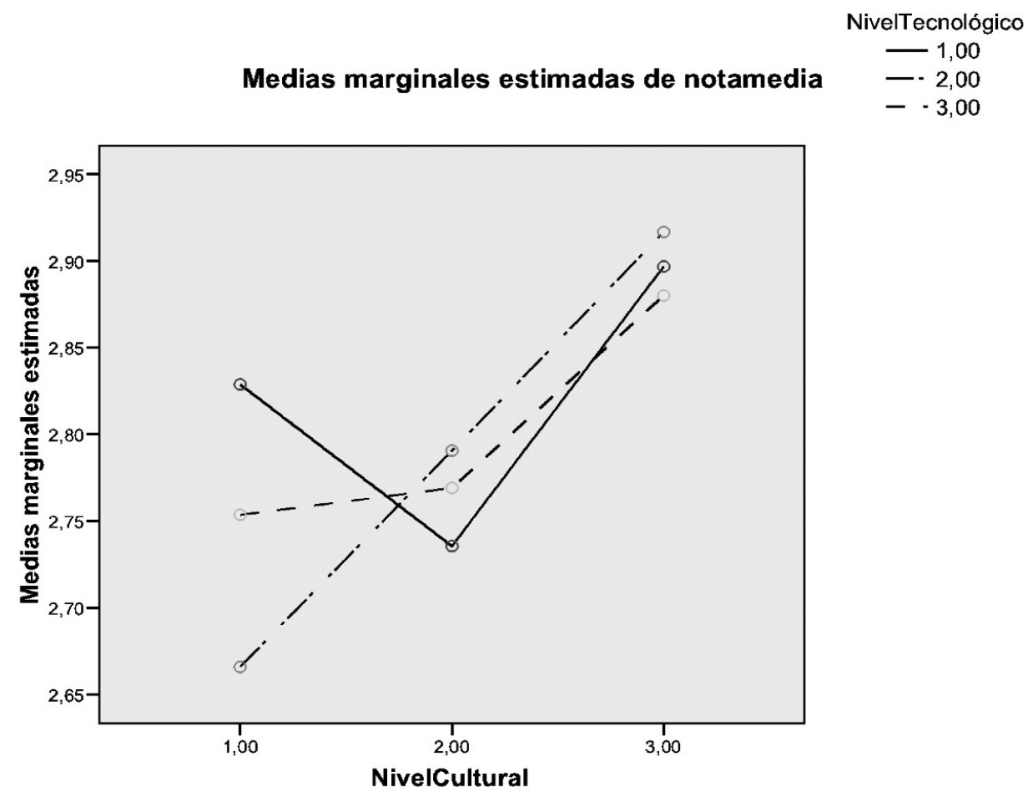

Gráfica 1. Interacción del ocio cultural y el ocio tecnológico sobre el rendimiento

Respecto a la lectura, casi la mitad de los estudiantes se sitúa en las categorías no lectoras: un $20.2 \%$ como no lector y un $27.1 \%$ en la categoría de falso lector. La mayoría, un $43.2 \%$ es lector ocasional y un $9.5 \%$ lector habitual.

El hábito lector resultó significativo sobre el rendimiento de los alumnos universitarios. Como puede observarse en la tabla 3, los estudiantes lectores obtienen en mayor porcentaje calificaciones más altas.

Tabla 3

Rendimiento según el hábito lector

\begin{tabular}{lcccccc}
\hline & No lector & $\begin{array}{c}\text { Falso } \\
\text { lector }\end{array}$ & $\begin{array}{c}\text { Lector } \\
\text { Ocasional }\end{array}$ & $\begin{array}{c}\text { Lector } \\
\text { Habitual }\end{array}$ & $\chi^{2}$ & $\boldsymbol{p}$ \\
\hline Suspenso & 1.2 & 0.3 & 0.6 & 0.5 & & \\
Aprobado & 26.8 & 22.7 & 16.6 & 18.3 & 14.51 & .002 \\
Notable & 68.8 & 74.1 & 78.2 & 73.3 & & \\
Sobresaliente & 3.2 & 2.8 & 4.7 & 7.9 & & \\
\hline
\end{tabular}


Los hábitos lectores correlacionan con el interés por la lectura literaria, $r=.594 . p<.001, \mathrm{y}$ también con el interés por la lectura profesional, $r=.128$. $p<.001$, pero no con la lectura de actualidad, $r=.046 . p=.104$. El interés por la lectura profesional incide significativamente sobre el rendimiento universitario (tabla 4).

Tabla 4

Media de rendimiento según interés lector

\begin{tabular}{lcccccc}
\hline & $\begin{array}{c}\text { Bajo } \\
\text { (hasta 5) }\end{array}$ & $\begin{array}{c}\text { Medio } \\
(\mathbf{d e ~ 6 ~ a ~ 8 ) ~}\end{array}$ & $\begin{array}{c}\text { Alto } \\
\mathbf{( 9 ~ y ~ 1 0 ) ~}\end{array}$ & $\boldsymbol{F}$ & $\boldsymbol{p}$ & $\eta^{\mathbf{2}}$ \\
\hline Profesional & 2.74 & 2.82 & 2.95 & 10.44 & .000 & .13 \\
Actualidad & 2.77 & 2.82 & 2.88 & 2.91 & .055 & .00 \\
Literaria & 2.77 & 2.85 & 2.85 & 2.87 & .057 & .00 \\
\hline
\end{tabular}

Nota: Escala de medida: 1-Suspenso, 2-Aprobado, 3-Notable, 4-Sobresaliente.

En último lugar, se analizaron las diferencias según el rendimiento en los dos conjuntos de variables de estudio, ocio y lectura, para intentar definir el perfil del estudiante universitario con rendimiento alto. Como la actividad de estudiar no entró en ninguno de los factores de ocio, la hemos incluido separadamente. Los resultados (tabla 5) indican que los alumnos con mejor rendimiento disponen de menos tiempo libre a diario y el fin de semana, aunque los estudiantes que suspenden y los que tienen aprobado dedican más tiempo libre a no hacer nada, sin diferencia significativa entre ellos, $p=.217$. Sin embargo, son los estudiantes con alto rendimiento los que se sienten más satisfechos con su tiempo de ocio, diferenciándose significativamente de los otros estudiantes (suspenso, $p<.001$, aprobado, $p<.05$ ), los alumnos que aprueban también se sientes significativamente más satisfechos que los alumnos que suspenden, $p<.001$. No se produce diferencia significativa respecto a ninguno de los otros factores de ocio. Se produce diferencia significativa en el tiempo que dedican al estudio, incrementándose significativamente conforme se incrementa la calificación. En las variables de lectura solamente el tiempo de lectura es similar en todos los estudiantes. En el número de libros leídos e interés por la lectura de actualidad, solo se diferencian significativamente los alumnos con media de aprobado de los alumnos con notable/sobresaliente (libros leídos, $p<.01$; interés lectura actualidad, $p<.001$ ). El interés por la lectura profesional es la variable con un tamaño mayor de efecto y se diferencia en los alumnos con mejor rendimiento respecto a los demás (suspenso, $p<.001$, aprobado, $p<.001$ ), el mismo resultado se produce en el interés por la lectura literaria (suspenso, $p<.05$, aprobado, $p<.05$ ). 
Tabla 5

Comparación de medias en las variables de ocio y lectura según rendimiento

\begin{tabular}{|c|c|c|c|c|c|}
\hline & Suspenso & Aprobado & $\begin{array}{c}\text { Notable/ } \\
\text { Sobresaliente }\end{array}$ & $\boldsymbol{F}$ & $\eta^{2}$ \\
\hline \multicolumn{6}{|l|}{ Ocio } \\
\hline Tiempo libre durante la semana ${ }^{1}$ & 3.08 & 2.80 & 2.60 & $14.06 * * *$ & .15 \\
\hline Tiempo libre el fin de semana ${ }^{1}$ & 3.51 & 3.36 & 3.24 & $3.57 *$ & .08 \\
\hline Ocio Social $^{1}$ & 3.02 & 3.18 & 3.12 & 1.70 & .02 \\
\hline Ocio Tecnológico ${ }^{1}$ & 3.17 & 3.16 & 3.12 & 0.41 & .02 \\
\hline Ocio Cultural $^{1}$ & 2.53 & 2.60 & 2.65 & 1.26 & .04 \\
\hline Ocio nada ${ }^{1}$ & 2.63 & 2.38 & 2.11 & $11.29 * * *$ & .13 \\
\hline Ocio Deporte ${ }^{1}$ & 2.54 & 2.68 & 2.65 & 0.23 & .01 \\
\hline Estudiar $^{1}$ & 2.65 & 3.25 & 3.42 & $16.73 * * *$ & .16 \\
\hline Satisfacción tiempo libre ${ }^{2}$ & 3.14 & 3.84 & 3.96 & $13.18 * * *$ & .14 \\
\hline \multicolumn{6}{|l|}{ Lectura } \\
\hline Tiempo de lectura ${ }^{3}$ & 2.88 & 2.77 & 2.92 & 1.81 & .05 \\
\hline Libros leídos ${ }^{4}$ & 2.23 & 1.80 & 1.99 & $3.82 *$ & .07 \\
\hline Interés lectura profesional ${ }^{5}$ & 6.17 & 6.64 & 7.40 & $31.49 * * *$ & .22 \\
\hline Interés lectura de actualidad ${ }^{5}$ & 7.29 & 7.14 & 7.59 & $9.25 * * *$ & .12 \\
\hline Interés lectura literaria ${ }^{5}$ & 6.47 & 6.42 & 6.70 & $3.88 *$ & .06 \\
\hline
\end{tabular}

Nota: 1: Escala de 1-Nada a 5-Mucho; 2: Escala de 1-Totalmente insatisfecho a 5-Totalmente satisfecho; 3: Escala de 0 -Nunca a 5-Todos los días; 4: Escala de 0 -Ninguno a 7-más de 50; 5: Escala de 1 a 10.

$* p<.05, * * p<.01, * * * p<.001$.

\section{DISCUSIÓN/CONCLUSIONES}

El objetivo de nuestro trabajo fue analizar la vinculación del ocio y de la lectura voluntaria con el rendimiento académico de los estudiantes universitarios. Nos hemos centrado en el enfoque de las potencialidades de los alumnos (Froh et al., 2011), aplicado al contexto universitario, para conocer el perfil de ocio y comportamiento lector de los estudiantes con alto rendimiento. Los resultados de esta investigación permitirán desarrollar programas de fortalecimiento del éxito en la formación universitaria.

En consonancia con otras investigaciones realizadas con estudiantes universitarios (Ureta, 2015), se ha encontrado un predominio del tiempo dedicado al ocio social. El que la actividad de estudiar no entrara en ningún factor del tiempo de ocio refuerza el concepto de ocio como una parte del tiempo libre. El tiempo libre se convierte en ocio cuando se emplea por libre elección para hacer lo que nos agrada. Para los estudiantes universitarios 
la actividad de estudiar es una necesidad, entra en obligatoriedad por su recorrido vital, estando fuera de la libre elección.

Los resultados confirmaron una incidencia del ocio cultural junto con el tecnológico en el rendimiento académico, aunque con un efecto reducido. Resultado congruente con la evolución del contexto de la universidad y de los universitarios, donde se valora más que nunca la Red como un espacio de aprendizaje (Gutiérrez-Perlán, Román-García, y Sánchez-Vera, 2018). La construcción del Espacio Europeo de Educación Superior ha supuesto una renovación metodológica donde las TIC han tomado preponderancia en la formación universitaria (Gutiérrez, Palacios, y Torrego, 2010). En la universidad los materiales se presentan en archivos digitales, las aulas pasan a plataformas virtuales y las actividades se realizan on-line. Respecto al comportamiento lector, en consonancia con estudios previos, nuestros resultados indican que el hábito lector se relaciona con la obtención de mejores calificaciones (Clerici et al., 2015; Lotti-de-Santos et al., 2008; Mol \& Jolles, 2014; Noursi, 2014; Whitten et al., 2016; Yubero, Larrañaga, y Sánchez-García, 2010). También nuestros resultados reflejan que a mayor tendencia a leer por placer - mayor hábito lector- se produce un mayor rendimiento académico en el contexto universitario.

Los estudiantes con un rendimiento de notable/sobresaliente se diferencian de los demás alumnos en estudiar más, lo que les lleva a disponer de menos tiempo libre a diario y el fin de semana. Aprovechan más su tiempo libre, dedicando menos tiempo a no hacer nada, mostrando una elevada satisfacción de su tiempo de ocio. También leen más libros y tienen mayor interés por la lectura.

En el perfil de los estudiantes con alto rendimiento la mayor diferencia se encuentra en el interés por la lectura profesional, aunque existen también diferencias en el interés por la lectura de actualidad y la lectura literaria. Sin embargo, el tiempo dedicado a la lectura es similar en todos los estudiantes. Debemos entender este resultado en el sentido de que los estudiantes tienen la necesidad de leer materiales de estudio, aparte de los de lectura voluntaria; buena parte del índice lector de los estudiantes universitarios se refiere a una lectura instrumental (Larrañaga, Yubero, y Cerrillo, 2008; Yubero et al., 2014). Los jóvenes universitarios en formación utilizan la lectura en sus actividades cotidianas de aprendizaje, en la búsqueda del conocimiento; es un eje fundamental en la cognición (Vidal y Manríquez, 2016). Por ello, hay un factor importante de sesgo de buena imagen vinculada por el comportamiento lector, ya encontrado en investigaciones previas (Larrañaga et al., 2008; Yubero et al., 2014), que lleva a que más de la cuarta parte se encuentre en la categoría de falsos lectores. 
Nuestros resultados indican que la lectura es una opción personal voluntaria que no depende de la disponibilidad del tiempo libre, en contraposición con lo que señalaban Rodríguez-Suárez y Agulló (1999). Los estudiantes con menor rendimiento disponen de más tiempo libre y no realizan ninguna actividad durante su tiempo de ocio, tampoco la lectura.

Aunque los estudiantes con alto rendimiento informan que disponen de menos tiempo libre durante la semana y el fin de semana, informan de mayor satisfacción con el mismo. Investigaciones previas ya habían señalado que no hacer nada, el ocio ausente, se relaciona con insatisfacción en el ocio (Lema, 2011). Por otra parte, como afirma Caride (2014), lo importante de las actividades de ocio es su significación para el desarrollo personal y social, y su contribución al bienestar, además de constituir experiencias satisfactorias (Cuenca, 2009) que deriven en un ocio valioso (Cuenca, 2013). Parece que, efectivamente, los alumnos con mejor rendimiento valoran positivamente dedicar su tiempo al estudio y la lectura, lo que les lleva a obtener buenas notas y mayor satisfacción de su tiempo libre. Tengamos en cuenta que no se diferencian en el ocio social, también interaccionan con sus amigos y disfrutan de su red social.

Mol y Jolles (2014) ya habían sugerido que se produce una espiral entre el placer por la lectura, la lectura voluntaria y el rendimiento. Esa misma espiral parece producirse en los estudiantes universitarios. Las diferencias relacionadas con el rendimiento se reflejan en el mayor interés lector, básicamente la lectura profesional, debido al itinerario formativo que están recorriendo (Cuenca y Goytia, 2012). Según reflejan los resultados, el hábito lector incide sobre los resultados académicos, pero es a través del placer por la lectura, concretado en el interés por la lectura profesional, donde se encuentran las diferencias entre los alumnos con bajo/normal rendimiento y los que han obtenido alto rendimiento. Estos resultados confirman la influencia positiva de la afición a la lectura sobre el rendimiento en el estudio (Tirado et al., 2010). Carlino (2006) ya había indicado que la lectura favorece la cultura discursiva de las disciplinas, aprendiendo los modos propios de leer y escribir en cada una de ellas. Diversos estudios con universitarios han indicado falta de conocimiento del vocabulario específico de su especialidad con explicaciones generales y poco técnicas en sus trabajos, ausencia de mecanismos intertextuales y pobre desempeño de comprensión lectora (Concha, Hernández, Del-Río, Romo, y Andrade, 2013; Neira, Reyes, y Riffo, 2015; Soto, 2009). Resultados que podrían deberse al bajo índice de lectores habituales entre los estudiantes universitarios, no llega al 10\%. Sin duda, la lectura externa a lo estrictamente curricular mejora la formación. La competencia literaria, explican Cerrillo y Sánchez-Ortiz (2017) — competencia lectora, añadimos nosotros-, lleva a que los lectores interactúen con el texto y que a partir de esa relación puedan plantearse 
preguntas, cuestionar con juicio propio, reflexionar sobre sí mismos y los demás a través de los personajes, identificarse con sus emociones y con los problemas planteados en la narrativa; por eso, incrementa la capacidad de interpretar la realidad. Esto podría ser la base de las diferencias encontradas en estudios previos en las intervenciones de discusiones (Lei et al., 2010; Sappington, Kinsey \& Munsayac, 2002; Torres, 2003) y con aportaciones más estimulantes (Ruscio, 2001).

La sociedad del conocimiento y sus instituciones, entre ellas también la universidad, deberían procurar una mejora de los hábitos lectores. Además, la lectura vinculada con el campo profesional facilitará la incorporación a la comunidad discursiva de la disciplina en la que se están formando. En conclusión, entre las potencialidades del alumno universitario podríamos incluir los hábitos lectores como trampolín hacia la lectura profesional.

A partir de estas conclusiones se deriva la importancia de diseñar propuestas de intervención en la universidad (y en etapas educativas previas) centradas en la mejora de las habilidades de lectura. Aunque se considera que las capacidades lectoras se han debido desarrollar en los niveles educativos anteriores (Benavides y Sierra, 2013) y que se pueden transferir sin dificultad entre los diferentes espacios académicos (González y Vega, 2010), parece preciso continuar potenciando el desarrollo y la consolidación de los hábitos lectores en el contexto universitario.

Como afirman Ricoy y Fernández-Rodríguez (2016), el centro educativo es clave para reforzar las actividades de ocio. Las universidades ofrecen a sus estudiantes importantes oportunidades para el tiempo libre poniendo a su disposición actividades, equipamientos e infraestructuras como complemento de la formación académica. La universidad, como institución educativa, debe fortalecer la importancia que tienen la lectura y la escritura en la formación académica de los estudiantes. Tal y como indican Sánchez-García y Yubero (2015), dentro de las competencias transversales y en los programas de actividades culturales, la universidad debería generar espacios de lectura dinámicos, flexibles y participativos que generen el desarrollo del comportamiento lector para conseguir que en el ambiente universitario se implante la cultura por la lectura, por supuesto, de los diversos tipos de textos y en diferentes soportes.

Entre las limitaciones del trabajo cabe señalar que, aunque el tamaño de la muestra es amplio, los datos se obtuvieron por conveniencia a través de profesores colaboradores. Además, se utilizó una medida de autoinforme, lo cual puede suponer un sesgo por el efecto de la deseabilidad social. Aunque para valorar el rendimiento pedimos a los alumnos la información sobre la nota media, cabe pensar que puedan existir también 'falsos rendimientos 
medios'. Las estimaciones generosas de su rendimiento medio podrían influir negativamente en la validez de los resultados obtenidos y, por tanto, en las conclusiones y propuestas vinculadas a los resultados. Por todo ello, sería interesante replicar la investigación a partir de datos reales. También resultarían necesarios estudios de carácter longitudinal que puedan especificar la direccionalidad entre las variables de estudio. En futuras investigaciones sería interesante analizar cómo afectan estas variables en función de los diferentes grados que cursen los estudiantes, así como completar los análisis añadiendo su relación con el bienestar personal y la adaptación a la universidad. También sería relevante poder conocer cómo influyen en la futura inserción en el mundo laboral. 


\section{REFERENCIAS BIBLIOGRÁFICAS}

Aristegui, I. y Silvestre, M. (2012). El ocio como valor en la sociedad actual. Arbor, 188, 283-291. doi: http://dx.doi. org/10.3989/arbor.2012.754n2001

Belvis, E., Moreno, M.V. y Ferrer, F. (2009). Los factores explicativos del éxito y fracaso académico en las universidades españolas, en los años del cambio hacia la convergencia europea. Revista Española de Educación Comparada, 15, 61-92.

Benavides, D.R. y Sierra, G.M. (2013). Estrategias didácticas para fomentar la lectura crítica desde la perspectiva de la transversalidad. Revista Iberoamericana sobre Calidad, Eficacia y Cambio en Educación, 11(3), 79-109.

Bermúdez, S., Ramírez, M.A. y Jaramillo, A. (2016). Preferencias en el uso del tiempo libre de los estudiantes universitarios con enfoque en el sector turístico. Revista CEA, 2(4), 75-88.

Bethencourt, J.T., Cabrera, L., Hernández, J.A., Álvarez, P., y González, M. (2008). Variables psicológicas y educativas en el abandono universitario. Revista Electrónica de Investigación Psicoeducativa, 6, 603-622.

Caride, J.A. (1998). Educación del ocio y del tiempo libre. En M. Beas y J. García (Coords.), Atención a los espacios y tiempos escolares (pp. 17-31). Granada: Grupo Editorial Universitario.

Caride, J.A. (2012). Lo que el tiempo educa: el ocio como construcción pedagógica y social. Arbor, 188 (754), 301-313. doi: http://dx.doi.org/ 10.3989/arbor.2012.754n2004

Caride, J.A. (2014). Del ocio como educación social a la pedagogía del ocio en el desarrollo humano.
Edetania: estudios y propuestas socioeducativas, 45, 33-53.

Caride, J.A. (Coord.) (2009). Los derechos humanos en la educación y la cultura: del discurso político a las prácticas educativas. Rosario-Argentina: Homo Sapiens.

Carlino, P. (2006). Escribir, leer y aprender en la universidad. Una introducción a la alfabetización académica. Buenos Aires: Fondo de Cultura Económica.

Carlino, P. (2013). Alfabetización académica 10 años después. Revista Mexicana de Investigación Educativa, 18(57), 355-381.

Centro Nacional de Información y Comunicación Educativa (2007). El ocio y los jóvenes. La web del estudiante. Ministerio de Educación y Ciencia. Recuperado de https://goo.gl/jAA9tE

Cerrillo, P.C. y Sánchez-Ortiz, C. (2017). Educación y competencia literarias (sobre la formación del lector literario). Revista Literatura em Debate, 11(21), 6-19.

Clark, C. \& Rumblod, K. (2006). Reading for Pleasure: A Research Overview. Londres, Reino Unido: National Literacy Trust

Clerici, C., Monteverde, A.C., y Fernández, A. (2015). Lectura, escritura y rendimiento académico en ingresantes universitarios. Ciencia, Docencia y Tecnología, 26, 35-70.

Concha, S., Hernández, C., Del-Río, F., Romo, F., y Andrade, L. (2013). Reflexión pedagógica en base a casos y dominio de lenguaje académico en estudiantes de cuarto año de pedagogía en educación básica. Calidad en la Educación, 38, 81-113. 
doi: http://dx.doi.org/10.4067/S071845652013000100002

Cuenca, M. y Goytia, A. (2012). Ocio experiencial: antecedentes y características. Arbor, 188, 265281. doi: http://doi.org/10.3989/ arbor.2012.754n2001

Cuenca, M. (2009). Ocio humanista. Dimensiones y manifestaciones actuales del ocio. Bilbao: Universidad de Deusto.

Cuenca, M. (2013). Ocio valioso en tiempos de crisis. En S. Torío et al. (Coords.), La crisis social y el Estado de Bienestar: las respuestas de la Pedagogía social (pp. 5-20). Oviedo: Servicio de Publicaciones de la Universidad de Oviedo.

Delors, J. (1997). La educación encierra un tesoro. Madrid: SantillanaEdiciones Unesco.

Dezcallar, T., Clariana, M., Cladelles, R., Badia, M., y Gotzens, C. (2014). La lectura por placer: su incidencia en el rendimiento académico, las horas de televisión y las horas de videojuegos. Ocnos, 12, 107-116. doi: http://dx.doi. org/10.18239/ocnos_2014.12.05

Dumazedier, J. (1971). Realidades del ocio e ideologías. En J. Dumazedier et al., Ocio y sociedad de clases (pp. 9-45). Barcelona: Fontanella.

Fabiano, F.M., Mckinney, G.K., Rhoads, K., \& Starks, C.H. (2000). WWU Lifestyles proyect IV. Washington, Estados Unidos: Washington University.

Fernández, N. (2005). La lectura entre las prácticas culturales de los jóvenes en España. Revista de Estudios de Juventud, 70, 13-21.

Fiz, M.R., Goicoechea, M.J., Ibiricu, O., y Olea, M.J. (2000). La comprensión lectora y su repercusión en el rendimiento escolar. Filología y Didáctica de la Lengua, 6-7, 75-108.

Froh, J.J., Huebner, E S., Youssef, A., \& Conte, V. (2011). Acknowledging and appreciating the full spectrum of the human condition: School psychology's (limited) focus on positive psychological functioning. Psychology in the Schools, 48(2), 110-123. doi: http://doi.org/10.1002/pits.20530

Galicia, J.C. y Villuendas, E.R. (2011). Relación entre hábitos lectores, el uso de la biblioteca y el rendimiento en una muestra de estudiantes de psicología. Revista de la Educación Superior, 157, 55-73.

García-Cué, J.L. y Santizo, J.A. (2010). Análisis de la relación entre la gestión del tiempo libre, el ocio y los estilos de aprendizaje. Revista Estilos de Aprendizaje, 5(5), 2-24.

García-Ros, R. y Pérez-González, F. (2011). Validez predictiva e incremental de las habilidades de autorregulación sobre el éxito académico en la universidad. Revista de Psicodidáctica, 16(2), 231-250.

Gil-Flores, J. (2011). Hábitos lectores y competencias básicas en el alumnado de educación secundaria obligatoria. Educación XXI, 14(1), 117-134.

González, B. y Vega, V. (2010). Prácticas de lectura y escritura en la universidad. Bogotá, Colombia: Fondo de Publicaciones de la Universidad Sergio Arboleda.

Gutiérrez, A., Palacios, A., y Torrego, L. (2010). Tribus digitales en las aulas universitarias. Comunicar, 17, 173181. doi: https://doi.org/10.3916/C342010-03-17

Gutiérrez-Perlán, I., Román-García, M., y Sánchez-Vera, M.M. (2018). Estrategias para la comunicación 
colaborativa en red de los estudiantes universitarios. Comunicar, 54, 91-100. doi: https://doi.org/10.3916/C54-201809

Hoeft, M.E. (2012). Why university students don't read: What professors can do to increase compliance. International Journal for the Scholarship of Teaching and Learning, 6(2). doi: https://doi.org/10.20429/ ijsotl.2012.060212

Kitsantas, A., Winsler, A., \& Huie, F. (2008). Self-regulation and ability predictors of academic success during college: A predictive validity study. Journal of Advanced Academics, 20 , 42-68.

Larrañaga, E. y Yubero, S. (2005). El hábito lector como actitud. El origen de la categoría de falsos lectores. Ocnos, 1, 43-60. doi: http://dx.doi. org/10.18239/ocnos_2005.01.04

Larrañaga, E. (2004). La lectura en los estudiantes universitarios: variables psicosociales en la formación de los hábitos lectores. Cuenca: Universidad de Castilla-La Mancha.

Larrañaga, E., Yubero, S., y Cerrillo, P. C. (2008). Estudio sobre los hábitos de lectura de los universitarios españoles. Madrid: CEPLI/SM.

Lassibille, G. \& Navarro, L. (2007). Why do higher education students drop out? Evidences from Spain. Education Economics, 16(1), 89-105. doi: https:// doi.org/10.1080/09645290701523267

Lei, S.A., Bartlett, K A., Gorney, S.E., \& Herschbach, T.R. (2010). Resistance to reading compliance among college students: Instructors' perspectives. College Student Journal, 44(2), 219229.

Lema, R. (2011). La recreación educativa: modelos, agentes y ámbitos. Revista
Latinoamericana de Recreación, 1, 7790.

Lotti-de-Santos, M., Salim, R, Raya, F., y Dori, M.G. (2008). Una experiencia de formación docente sobre la lectura comprensiva de textos científicos. Revista Iberoamericana de Educación, 5. Recuperado de https://goo.gl/2855sU

Mol, S.E. \& Jolles, J. (2014). Reading enjoyment amongst non-leisure readers can affect achievement in secondary school. Frontiers in Psychology, 5. doi: http://doi. org/10.3389/fpsyg.2014.01214

Molina, L. (2006). Lectura y Educación: los hábitos lectores y su repercusión académica en la ESO. Ocnos, 2, 103120. doi: http://dx.doi.org/10.18239/ ocnos_2006.02.07

Moyano, E. (2004). La escritura académica: una tarea interdisciplinaria a lo largo del currículo universitario. Revista Textura, 4(4), 109-120.

Neira, A., Reyes, F., \& Riffo, B. (2015). Academic experience and reading comprehension strategies in Frehmen University students. Literatura y Lingüística, 31, 221-244. doi: http://dx.doi.org/10.4067/S071658112015000100012

Noursi, O.A. (2014). Teaching comprehension: What teachers should know? Perspectives, 22(1), 11-22.

Organización para la Cooperación y el Desarrollo Económicos (2012). Programme for International Students Assessment, PISA, 2011. Madrid: Ministerio de Educación, Cultura y Deporte (MECD).

Poza-Vilches, F., Fernández-García, A., y Ferreira-Delgado, J.P. (2018). La práctica profesional de los agentes sociales en materia de ocio juvenil: estrategias para la 
intervención, Pedagogía Social. Revista Interuniversitaria, 31, 93105. doi: http://dx.doi.org/10.7179/ PSRI_2018.31.08

Ricoy, M.C., y Fernández-Rodríguez, J. (2016). Prácticas y recursos de ocio en la adolescencia. Educatio Siglo XXI, 34(2), 103-124. doi: http://dx.doi. org $/ 10.6018 / \mathrm{j} / 263831$

Rodríguez-Suárez, J., y Agulló, T. (1999). Estilos de vida, cultura, ocio y tiempo libre de los estudiantes universitarios. Psicothema, 11(2), 247-259.

Ruscio, J. (2001). Administering quizzes at random to increase students' reading. Teaching of Psychology, 28, 204-206. doi: http://dx.doi.org/10.1207/ S15328023TOP2803_08

Sánchez-Chévez, L.E. (2012). La comprensión lectora en el currículo universitario. Diálogos, 9, 21-36.

Sánchez-García, S. \& Yubero, S. (2015) Leer en la universidad: la promoción de la lectura desde la biblioteca universitaria. En S. Sánchez-García \& S. Yubero, Las bibliotecas en la formación del hábito lector (pp. 159188). Cuenca: Servicio Publicaciones UCLM.

Sappington, J., Kinsey, K., \& Munsayac, K. (2002). Two studies of reading compliance among college students. Teaching of Psychology, 29(4), 272274. doi: https://doi.org/10.1207/ S15328023TOP2904_02

Soto, G. (2009). Intertextualidad explícita en textos académicos de estudiantes universitarios: Un estudio exploratorio. Literatura y Lingüística, 20, 141-157. doi: http://doi.org/10.4067/ S0716-58112009000100008

Tejedor, F.J. \& García-Valcárcel, A. (2007). Causas del bajo rendimiento del estudiante universitario (en opinión de los profesores y alumnos). Propuestas de mejora en el EEES. Revista de Educación, 342, 443-473.

Tirado, R., Aguaded, J.I., y Marín, I. (2010). Patrones de consumo de drogas y ocupación del ocio en estudiantes universitarios. Sus efectos sobre el hábito de estudio. Revista Española de Drogodependencia, 35(4), 467-480.

Torres, E. (2003). Bases teóricas para la comprensión lectora, eficaz, creativa y autónoma. Educere, 6(20), 380-383.

Ureta, M.X. (2015). Estilos de vida y prácticas de ocio en estudiantes de la licenciatura de Educación Física, Recreación y Deporte de la IUACJ. Revista Universitaria de Educación Física y el Deporte, 8, 49-58.

Vidal, D. y Manriquez, L. (2016). El docente como mediador de la comprensión lectora en universitarios. Revista de la Educación Superior, 177, 95-118.

Whitten, C., Labby, S., \& Sullivan, S.L. (2016). The impact of pleasure reading on academic success. The Journal of Multidisciplinary Graduate Research, 2, 48-64.

Yubero, S. y Larrañaga, E. (2015). Lectura y universidad: estudio de los hábitos lectores de los estudiantes universitarios de España y Portugal. El Profesional de la Información, 24(6), 713-719. doi: http:/dx.doi.org/10.3145/ epi.2015.nov.03

Yubero, S., Larrañaga, E., e Pires, N. (2014). Estudo sobre os hábitos de leitura dos estudantes protugueses do ensino superior. Castelo Branco, Portugal: Instituto Politécnico de Castelo Branco.

Yubero, S., Larrañaga, E., y SánchezGarcía, S. (2010). El valor de la lectura y su relación con los hábitos lectores y 
el éxito escolar en niños inmigrantes. Educar Educere, número especial, 5163.

Yubero, S., Larrañaga, E. y SánchezGarcía, S. (2011). El valor de la lectura y su relación con el comportamiento lector y el éxito escolar en niños de Primaria. En A.M. Ramos \& I. Mociño (Eds.), Crítica e investigación en LIJ (pp. 345-356). Vigo/Braga: ANILIJ/ Universidad de Minho. 


\section{PERFIL ACADÉMICO Y PROFESIONAL DE LOS AUTORES}

María Elche Larrañaga. Graduada en educación social, es investigadora predoctoral de la Universidad de Castilla-La Mancha. Posee el título propio «Leer y vivir la igualdad. Educar actitudes igualitarias desde la lectura» del Centro de Estudios de Promoción de la Lectura y la Literatura Infantil (Cepli) y el «Máster Universitario de Investigación en Psicología Aplicada», además del «VIII Máster de Promoción de la Lectura y Literatura Infantil». Ha publicado distintos trabajos y participado en diversos congresos nacionales e internacionales.

Sandra Sánchez García. Diplomada en biblioteconomía, licenciada en documentación y doctora en la filología hispánica y clásica, es directora de la Biblioteca General del Campus de Cuenca, de la Universidad de Castilla-La Mancha y profesora asociada en la Facultad de Ciencias de la Educación y Humanidades de esta misma universidad. Es secretaria técnica del CEPLI (Centro de Estudios de Promoción de la Lectura y Literatura Infantil) y coordinadora editorial de Ocnos: Revista de estudios sobre lectura.

Santiago Yubero Jiménez. Doctor en psicología y licenciado en pedagogía, es catedrático de escuela universitaria de psicología social de la educación. Es decano de la Facultad de Ciencias de la Educación y Humanidades, subdirector del Centro de Estudios para la Promoción de la Lectura y Literatura Infantil (Cepli) de la Universidad de Castilla-La Mancha y codirector de la revista Ocnos. Coordina el grupo de investigación Psicología, Educación y Lectura (PEL).

Dirección de los autores: Universidad de Castilla-La Mancha

Facultad de Ciencias de la Educación y Humanidades

Avda de los Alfares, 42

16071 Cuenca

E-mail: maria.elche@uclm.es sandra.sanchez@uclm.es santiago.yubero@uclm.es

Fecha recepción del artículo: 08. Marzo. 2018

Fecha modificación del artículo: 27. Junio. 2018

Fecha aceptación del artículo: 30. Junio. 2018

Fecha revisión para publicación: 20. Junio. 2018 